\title{
Therapeutic effect of low-molecular-weight heparin on adult sepsis: a meta-analysis
}

\author{
Xinyu Li", Zhengxiong Liu\#, Menghua Luo, Yonglin Xi, Cheng Li, Shuixian Wang, Rong Yang \\ Department of Critical Medicine, the Second Affiliated Hospital of Xi'an Medical University, Xi'an, China \\ Contributions: (I) Conception and design: X Li, Z Liu; (II) Administrative support: X Li; (III) Provision of study materials or patients: C Li, S Wang, \\ R Yang; (IV) Collection and assembly of data: M Luo, Y Xi; (V) Data analysis and interpretation: X Li, Z Liu; (VI) Manuscript writing: All authors; (VII) \\ Final approval of manuscript: All authors. \\ "These authors contributed equally to this work. \\ Correspondence to: Xinyu Li. Department of Critical Medicine, the Second Affiliated Hospital of Xi'an Medical University, No. 167 Fangdong Street, \\ Baqiao District, Xi'an 710000, China. Email: lxy_icu@163.com.
}

\begin{abstract}
Background Low-molecular-weight heparin (LMWH) is a part of standard supportive therapy for sepsis, but clinical research on anticoagulant therapy is still controversial. The aim of the present study was to explore the efficacy and safety of LMWH in adult septic patients by meta-analysis.

Methods: Information on randomized controlled trials was retrieved from PubMed, Embase, Web of Science, China National Knowledge Infrastructure, and China Wanfang Database from the establishment of each database to February 20, 2020. The therapeutic efficacy indexes of LMWH for adult septic patients were analyzed by Stata15.0 software.

Results: A total of 684 septic patients were included in 10 trials. The results showed that, compared with conventional treatment, LMWH significantly reduced prothrombin time [mean difference $(\mathrm{MD})=-0.48$, 95\% confidence interval (CI): -0.83 to -0.13 ], Acute Physiology And Chronic Health Evaluation II score $(\mathrm{MD}=-4.42,95 \% \mathrm{CI}:-5.50$ to -3.33$)$, and 28 -day mortality [relative risk $(\mathrm{RR})=0.52,95 \% \mathrm{CI}: 0.38-0.70$ ], and increased platelet count $(\mathrm{MD}=34.99,95 \% \mathrm{CI}: 23.37-46.60)$. LMWH significantly reduced levels of tumor necrosis factor- $\alpha$, interleukin-6, and D-dimer, and the incidence of multiple organ dysfunction syndrome (MODS), with statistically significant differences. In addition, LMWH did not increase bleeding events (RR $=1.29,95 \%$ CI: $0.76-2.17$ ).
\end{abstract}

Conclusions: On the basis of routine treatment, LMWH can improve coagulation function, reduce inflammatory reaction and the risk of bleeding, reduce the incidence of MODS and 28-day mortality rate, and improve the prognosis of adult patients with sepsis.

Keywords: Sepsis; low-molecular-weight heparin (LMWH); meta-analysis

Submitted Dec 24, 2020. Accepted for publication Mar 03, 2021.

doi: 10.21037/apm-21-169

View this article at: http://dx.doi.org/10.21037/apm-21-169

\section{Introduction}

Sepsis is a systemic infection with systemic inflammatory response syndrome caused by pathogenic microorganisms, especially bacteria. It is important that it is the main cause of death in intensive care unit (ICU) except cardiac diseases, and its patients account for $1.3 \%$ of the total number of hospitalized patients and $10 \%$ of the number of patients in the ICU $(1,2)$. After the invasion of bacteria, the hemostatic function of the human body is activated to resist infection and eventually forms thrombin and fibrin, both of which will further promote the occurrence of inflammation (3). In addition, fibrinolytic reaction is also inhibited. Excessive procoagulant and antifibrinolytic reactions may lead to coagulation disorders, such as thrombocytopenia, disseminated intravascular coagulation, microthrombosis 
and multiple organ dysfunction syndrome (MODS) (4). Therefore, to some extent, anticoagulant can be used as a potential therapeutic scheme for sepsis $(5,6)$. Studies have pointed out that heparin plays an anticoagulant role by enhancing the activity of antithrombin and inactivating $\mathrm{Xa}$ and IIa (thrombin) factors (7). Thrombin is closely related to inflammation, so the inhibition of thrombin is equivalent to the inhibition of inflammation $(8,9)$. Heparin is therefore considered to have an anti-inflammatory effect that is independent of its anticoagulant effect, and there is no correlation between the two (10). The Sepsis Surviving Campaign and Guidelines for Prevention of Venous Thromboembolism in Non-surgical Patients both recommend septic patients use unfractionated heparin (UFH) or low-molecular-weight heparin (LMWH) for thromboprophylaxis $(11,12)$. UFH has been used as an anticoagulant for more than 60 years, and has been found to significantly inhibit thrombin, and can inhibit Xa, IXa, $\mathrm{Xla}$, and Xlla coagulation factors through antithrombin (13). $\mathrm{LMWH}$ is an anticoagulant derived from UFH through chemical or enzymatic hydrolysis. Its anticoagulant effect, like UFH, is also based on activating antithrombin. The average molecular weight of $\mathrm{LMWH}$ is smaller than half of that of UFH, therefore it has little effect on thrombin. $\mathrm{LMWH}$ is mainly used to inactivate factor $\mathrm{Xa}$. The size of heparin will affect the interaction between heparin and platelets. It can enhance or inhibit platelet aggregation, which is one of its adverse properties and one of the causes of side-effects, including hemorrhage (14). Antibodies produced by UFH and platelet factor 4 (PF4) complexes can lead to heparin-induced thrombocytopenia (HIT). Because LMWH is relatively small, it also reduces its binding to platelets and $\mathrm{PF} 4$, therefore reducing the incidence of HIT (15). LMWH has also been found to increase fibrinolysis (16). Therefore, compared with UFH, $\mathrm{LMWH}$ is considered to be a more effective anticoagulant that not only retains good antithrombotic effect but also reduces the risk of bleeding, therefore reducing the risk of complications. At present, clinical research on anticoagulant therapy for sepsis is still controversial, and whether heparin or LMWH should be used for anticoagulant therapy for sepsis is still in doubt. Therefore, the aim of the present study was to comprehensively analyze previous research on the therapeutic effects of $\mathrm{LMWH}$ on sepsis so as to provide evidence-based medicine for the clinical anticoagulation treatment of sepsis.

We present the following article in accordance with the PRISMA reporting checklist (available at http://dx.doi. org/10.21037/apm-21-169).

\section{Methods}

\section{Literature retrieval}

Databases, including PubMed, Web of Science, Embase, China Wanfang Database, China National Knowledge Infrastructure, and Biomedical Literature Database of China, were searched to collect clinical randomized controlled trials of LMWH for sepsis patients. "Lowmolecular-weight heparin" and "sepsis" or "severe sepsis" or "septic shock" or "septic shock" were used as the keywords. The search time was from the establishment of each database to February 20, 2020. We used the references included in the literature to supplement the research that might have been missed. Two evaluators independently screened documents according to the steps of preliminary screening and full-text screening. Any differences were settled through discussions between both parties or judged with the assistance of a third party.

\section{Criteria for inclusion and exclusion of documents}

The inclusion criteria were as follows: (I) published prospective, randomized controlled clinical studies on LMWH treatment of sepsis, limited to English and Chinese; (II) diagnostic criteria conformed to the diagnostic criteria for sepsis, severe sepsis, and septic shock formulated by the American Society of Critical Care Medicine in 2001; (III) purpose of the study was to explore the effect of LMWH on the prognosis and adverse events of sepsis; and (IV) study participants were aged $>18$ years.

The exclusion criteria were as follows: (I) Semirandomized controlled trials or non-randomized controlled trials, animal experiments, mechanism studies, case reports, and reviews; (II) studies with insufficient data; (III) death or discharge within $72 \mathrm{~h}$ after treatment; (IV) the research object includes children; and (V) all clinical randomized controlled trials on LMWH treatment of sepsis, whether blinded or not, were included.

\section{Intervention measures and determination indicators of therapeutic effect}

The intervention methods were as follows: (I) the treatment group was treated with $\mathrm{LMWH}$, while the control group was treated with routine treatment; (II) the LMWH 


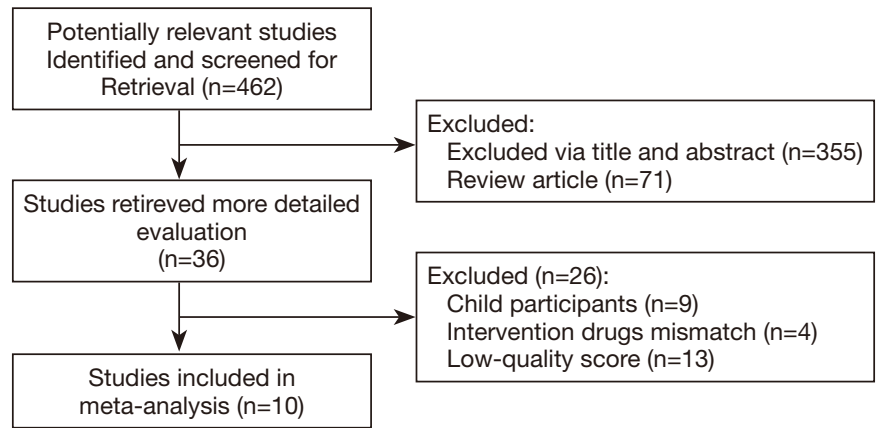

Figure 1 Article retrieval flow diagram.

treatment group was treated with LMWH; and (III) routine treatment included early antibiotics, fluid resuscitation, blood sugar control, organ support, and basic etiological support.

One or more of the following determination indicators of therapeutic effect were included: Acute Physiology And Chronic Health Evaluation II (APACHE II) score, D-dimer level, platelet count, prothrombin time (PT), 28-day mortality rate, MODS incidence rate, bleeding complications, tumor necrosis factor- $\alpha(\mathrm{TNF}-\alpha)$ and interleukin (IL)-6.

\section{Literature quality evaluation}

Methodological quality evaluation of the study adopts the method recommended by the Cochrane Collaboration Network. The quality evaluation of each study includes random allocation method; whether the allocation scheme is hidden; whether blind method is used; and whether to describe withdrawal and missing visits, and if so, whether an intentionality analysis should be conducted.

\section{Statistical methods}

Data were statistically analyzed by Stata 15.0 software (STATACORP LP, Texas City, USA). For quantitative data, the combined estimation was the mean difference (MD) and its $95 \%$ confidence interval (CI). For binary data, the combined estimation was the relative risk (RR) and its $95 \%$ CI. LMWH plus conventional therapy was compared with conventional therapy. Cochran's Q test and $\mathrm{I}^{2}$ statistics were used to evaluate the heterogeneity of the study. If $\mathrm{I}^{2} \geq 50 \%$ or $\mathrm{P}<0.05$, this indicated significant heterogeneity, and the random-effect model was selected for data merging (17); the fixed-effect model was used for data consolidation. Potential publication bias was assessed by funnel plot and Egger's test. Sensitivity analysis was conducted on the research results to evaluate the robustness of the conclusions.

\section{Results}

\section{Literature search results and baseline characteristics}

In total, 462 potential articles were initially retrieved, of which 426 were excluded after screening of titles and abstracts. After full-text retrieval, 26 articles were excluded for various reasons. Finally, 10 articles were included in the study (Figure 1) (18-27). Baseline characteristics included in the study are shown in Table 1. All tests were published in Chinese, but no research published in English was found. The 10 trials involved 684 patients, 363 of whom were in the LMWH group and 321 in the routine treatment group. The LMWH group received two types of heparin therapy: low-molecular heparin calcium and low-molecular heparin sodium. The treatment time of LMWH was 7 days. The included tests were classified as low to medium quality. Figure 2 summarizes the bias risk for each test.

\section{Meta-analysis results}

\section{Clinical outcomes}

The results of the meta-analysis are shown in Table 2. Nine articles reported APACHE II scoring results (Figure 3A). The random-effect model $\left(\mathrm{I}^{2}=60.9 \%, \mathrm{P}<0.05\right)$ was used for analysis. Compared with the conventional treatment group, the APACHE II score in the LMWH treatment group was significantly reduced ( $\mathrm{MD}=-4.42,95 \% \mathrm{CI}:-5.50$ to -3.33 ). Seven articles reported 28-day mortality. Using the fixedeffect model $\left(\mathrm{I}^{2}=0 \%, \mathrm{P}>0.05\right)$, LMWH treatment was found to significantly reduce the 28 -day mortality rate $(\mathrm{RR}=0.52$, 
Table 1 Basic characteristics of literature inclusion

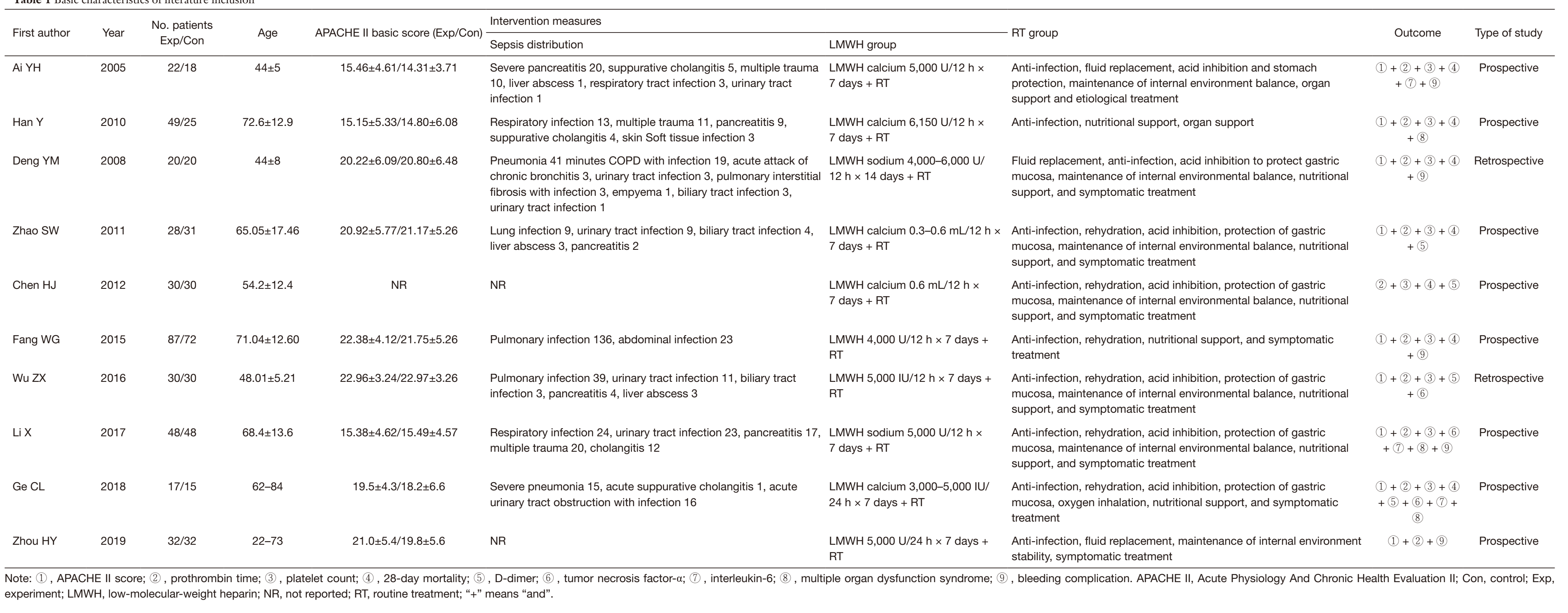




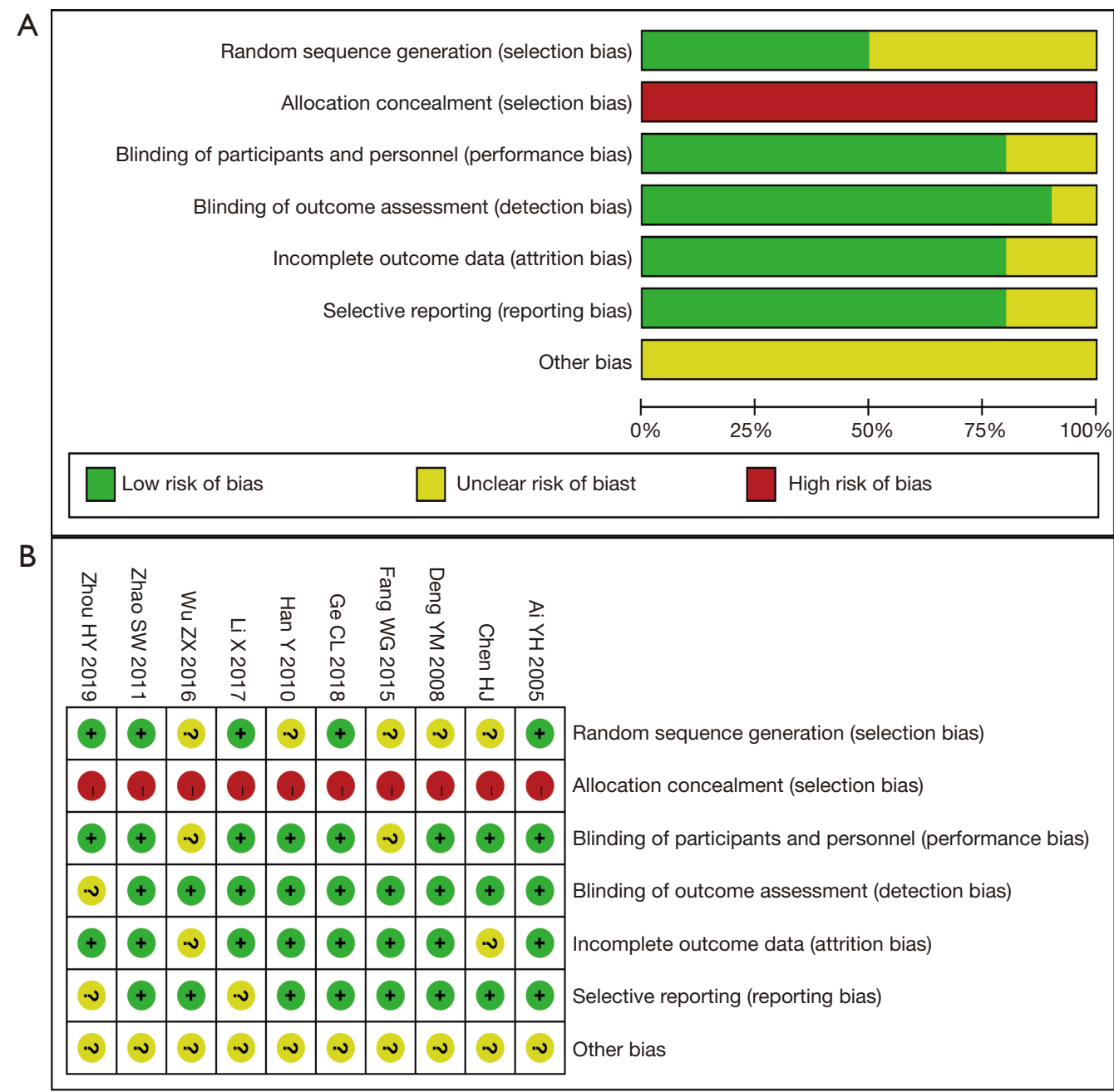

Figure 2 Results of literature quality risk assessment. (A) Risk of bias graph; (B) risk of bias summary.

95\% CI: 0.38-0.70) (Figure 3B).

\section{Anticoagulant activity}

Using fixed-effect model analysis $\left(\mathrm{I}^{2}=4.3 \%, \mathrm{P}>0.05\right)$, $\mathrm{LMWH}$ treatment was found to significantly reduce serum D-dimer (MD $=-266.91,95 \%$ CI: -339.98 to -193.85 ) (Figure 4A). Nine articles on platelet count were included. Using the random-effect model $\left(\mathrm{I}^{2}=54.9 \%, \mathrm{P}<0.05\right)$, platelet count (MD $=34.99,95 \%$ CI: 23.37 to 46.60) was found to be significantly higher than that of the conventional treatment group (Figure 4B). All studies on PT were included in our meta-analysis. Using the fixed-effect model $\left(\mathrm{I}^{2}=42.2 \%\right.$, $\mathrm{P}>0.05)$, LMWH treatment was found to significantly reduce PT ( $M D=-0.48,95 \%$ CI: -0.83 to -0.13$)$. Four articles on serum D-dimer were included (Figure 4C).

\section{Serum inflammatory factors}

Three articles on serum IL-6 levels were included (Figure $4 D)$. Using the random-effect model $\left(\mathrm{I}^{2}=54.9 \%\right.$, $\mathrm{P}<0.05)$, the serum IL-6 level in the LMWH treatment group was found to be significantly lower than that in the conventional treatment group $(\mathrm{MD}=-85.92,95 \% \mathrm{CI}$ : -148.78 to -23.06$)$. Three articles on serum TNF- $\alpha$ levels were included (Figure $4 E$ ). Using the random-effect model $\left(\mathrm{I}^{2}=92.2 \%, \mathrm{P}<0.05\right)$, serum TNF- $\alpha$ level in the LMWH treatment group was found to be significantly reduced $(\mathrm{MD}=-7.94,95 \% \mathrm{CI}:-13.07$ to -2.82$)$.

\section{Hemorrhagic complications and MODS incidence rate}

Five articles reported bleeding events (Figure $5 A$ ). Using the fixed-effect model $\left(\mathrm{I}^{2}=0 \%, \mathrm{P}>0.05\right)$, bleeding complications 
Table 2 Main results of meta-analysis

\begin{tabular}{|c|c|c|c|c|c|c|c|c|c|}
\hline Effect index & $\mathrm{n}$ & MD & $\mathrm{RR}$ & $95 \% \mathrm{Cl}$ & $P$ value & $\mathrm{I}^{2}(\%)$ & $P$ for $I^{2}$ & Model & $\mathrm{P}$ for publication bias \\
\hline PT & 10 & -0.48 & NA & -0.83 to -0.13 & 0.008 & 42.2 & 0.076 & FEM & 0.258 \\
\hline Platelets & 9 & 34.99 & NA & 23.37 to 46.60 & 0.000 & 54.9 & 0.023 & REM & 0.875 \\
\hline 28-day mortality & 7 & NA & 0.52 & 0.38 to 0.70 & 0.000 & 0.0 & 0.789 & FEM & 0.481 \\
\hline $\begin{array}{l}\text { Bleeding } \\
\text { complication }\end{array}$ & 5 & NA & 1.29 & 0.76 to 2.17 & 0.349 & 0.0 & 0.548 & FEM & 0.029 \\
\hline D-dimer & 4 & -266.91 & NA & -339.98 to -193.85 & 0.000 & 4.3 & 0.371 & FEM & 0.094 \\
\hline TNF- $\alpha$ & 3 & -7.94 & NA & -13.07 to -2.82 & 0.002 & 92.2 & 0.000 & REM & 0.994 \\
\hline
\end{tabular}

APACHE II, Acute Physiology And Chronic Health Evaluation II; FEM, fixed-effect model; MD, mean difference; MODS, multiple organ dysfunction syndrome; TNF- $\alpha$, tumor necrosis factor- $\alpha$; IL-6, interleukin-6; NA, not available; PT, prothrombin time; REM, random-effect model; RR, relative risk.
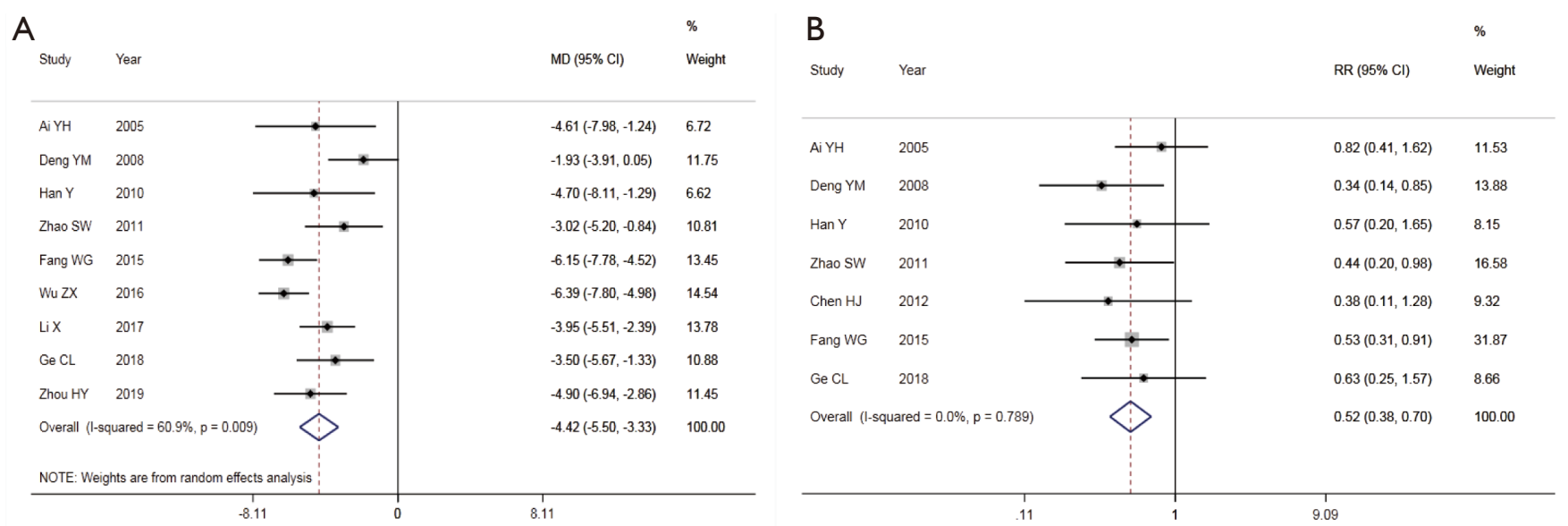

Figure 3 Forest plots of clinical outcomes. (A) Acute Physiology And Chronic Health Evaluation II score; (B) 28-day mortality rate. CI, confidence interval; $\mathrm{MD}$, mean difference; $\mathrm{RR}$, relative risk.

in the LMWH treatment group did not increase compared with the conventional treatment group ( $R R=1.29,95 \%$ CI: 0.76 to 2.17. The incidence of MODS was reported in 3 articles (Figure $5 B$ ). Using the fixed-effect model $\left(\mathrm{I}^{2}=0 \%\right.$, $\mathrm{P}>0.05$ ), the incidence of MODS in the LMWH treatment group was significantly lower than that in the conventional treatment group ( $\mathrm{RR}=0.54,95 \% \mathrm{CI}: 0.36$ to 0.80$)$.

\section{Publication bias}

The results of Egger's test of all indexes are shown in Table 2. Except for the test P values of IL-6 and bleeding complications were slightly less than 0.05 , other indicators were all $>0.05$, indicating that the difference was not statistically significant. The funnel plot of APACHE II, PT, platelet count, and 28-day mortality showed that the graphs of these four indicators were basically symmetrical (Figure 6). As the number of indicators is small, no funnel chart was created. Overall, the publication bias of the present study was relatively small.

\section{Sensitivity analysis}

The sensitivity analysis results the of APACHE II score, 
A

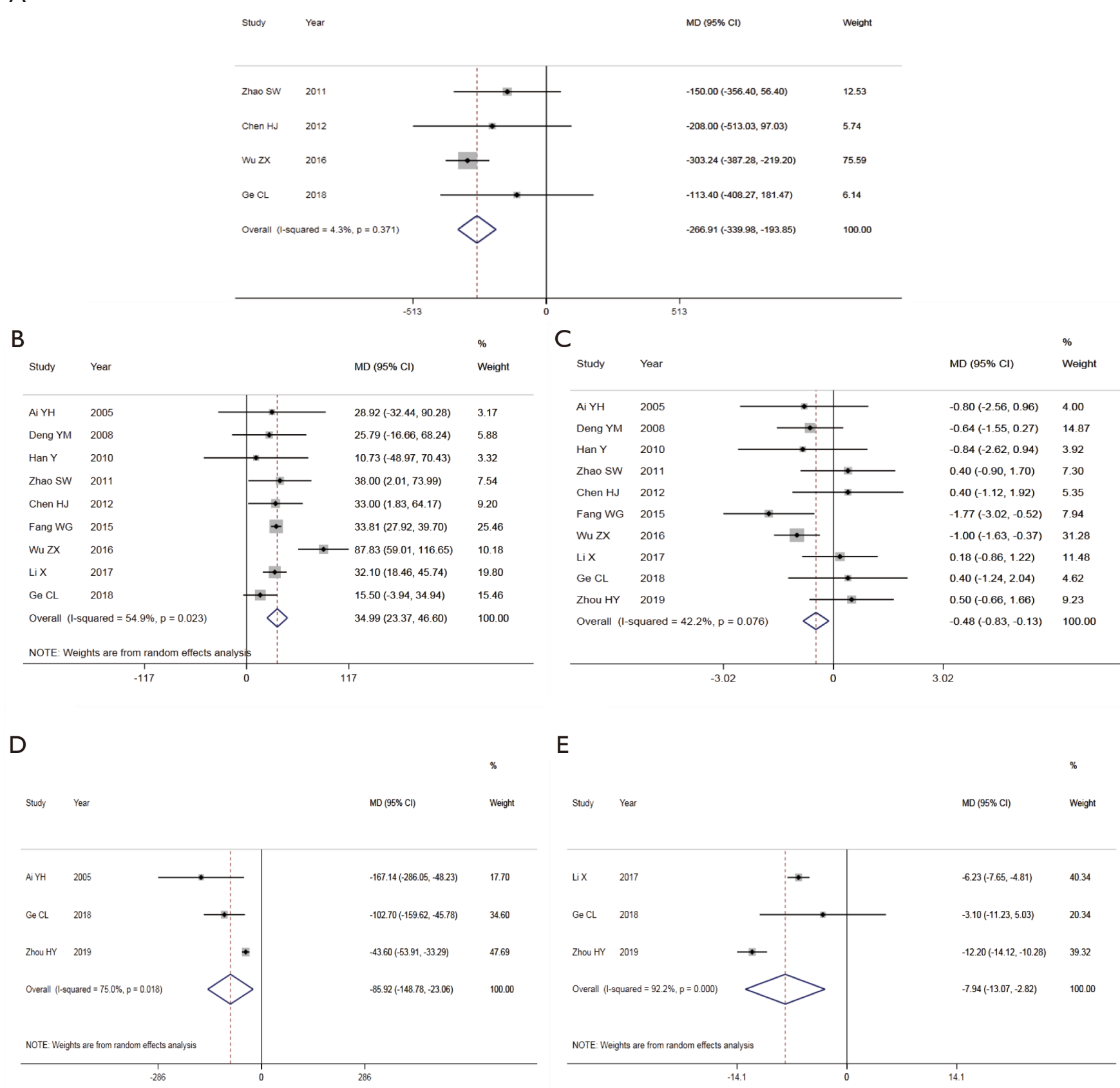

Figure 4 Forest plots of anticoagulant activity and serum inflammatory factors. (A) D-dimer; (B) platelet count; (C) prothrombin time; (D) serum interleukin-6 level; (E) serum tumor necrosis factor- $\alpha$ level. CI, confidence interval; MD, mean difference.

28-day mortality rate, hemorrhagic complications, and MODS showed that the results of the meta-analysis after excluding a single study had no statistically significant changes (Figure 7). Therefore, for these 4 indicators, the results were stable.
Sensitivity analysis results of platelet count, PT, D-dimer, TNF- $\alpha$, and IL- 6 showed that the results of the metaanalysis of platelet count and D-dimer after excluding 1 study were consistent with the original conclusion (Figure 8). However, after eliminating 2 articles, 1 article, and 1 article 
A

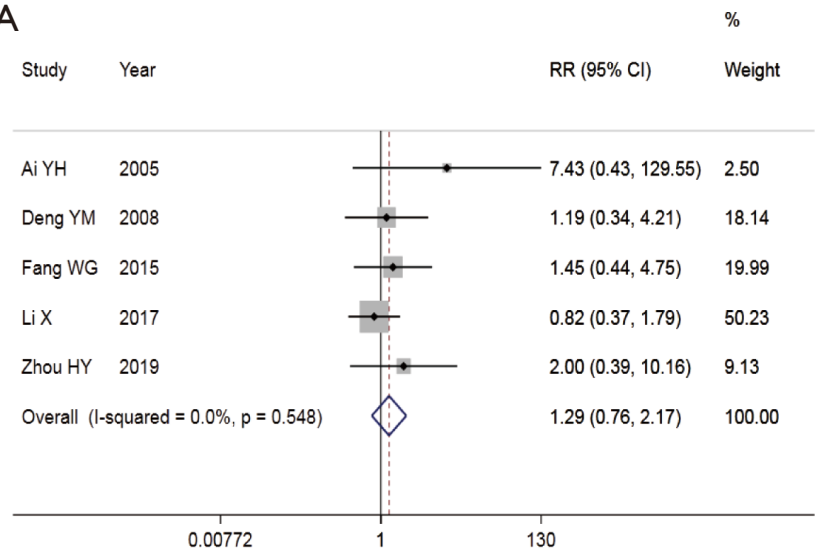

B

Study Year

Year

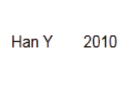

LiX $\quad 2017$

$\mathrm{Ge} C L \quad 2018$

Overall (I-squared $=0.0 \%, p=0.763$ )
RR $(95 \% \mathrm{Cl}) \quad$ Weight

$0.40(0.15,1.07) \quad 22.92$

$0.57(0.33,0.98) \quad 52.72$

$0.62(0.32,1.21) \quad 24.36$

$0.54(0.36,0.80) \quad 100.00$

Figure 5 Forest plots of hemorrhagic complications and multiple organ dysfunction syndrome incidence rate. (A) Hemorrhagic complications; (B) multiple organ dysfunction syndrome. CI, confidence interval; RR, relative risk.

A

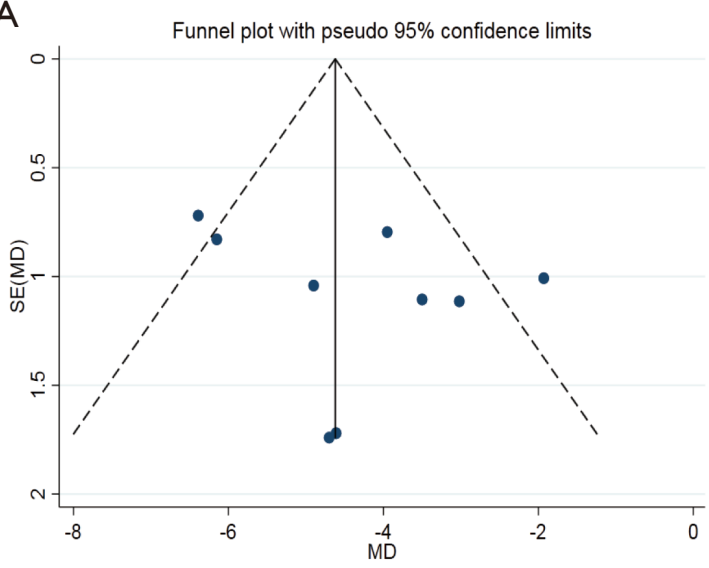

C

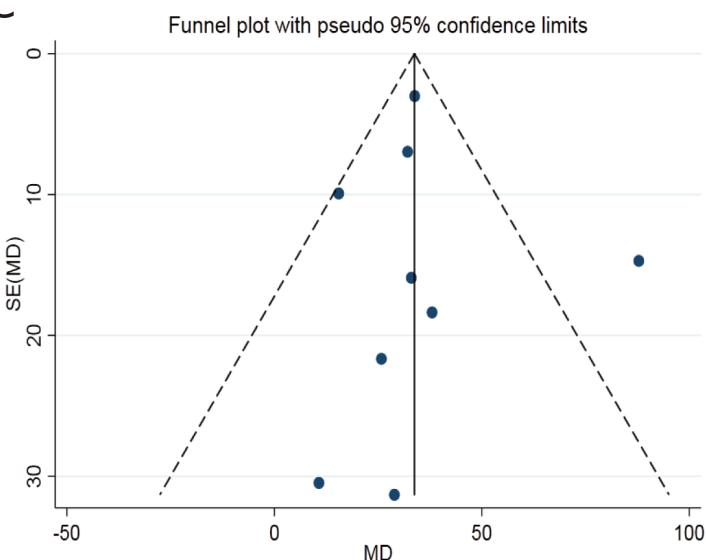

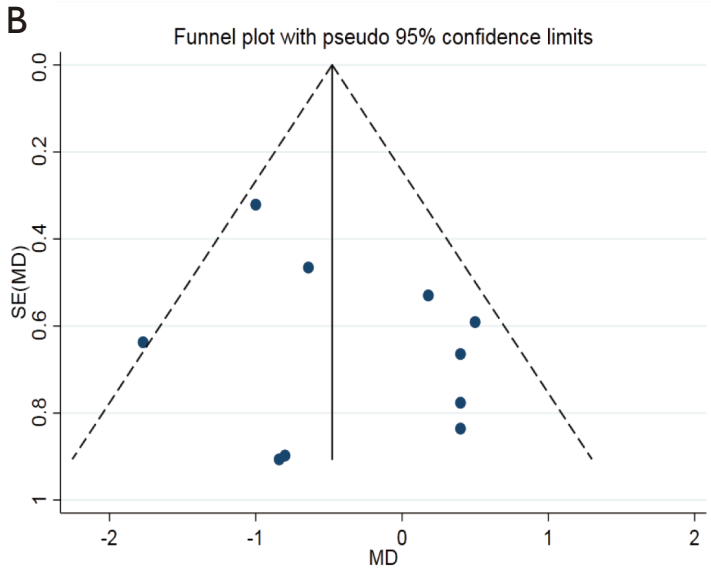

D Funnel plot with pseudo $95 \%$ confidence limits

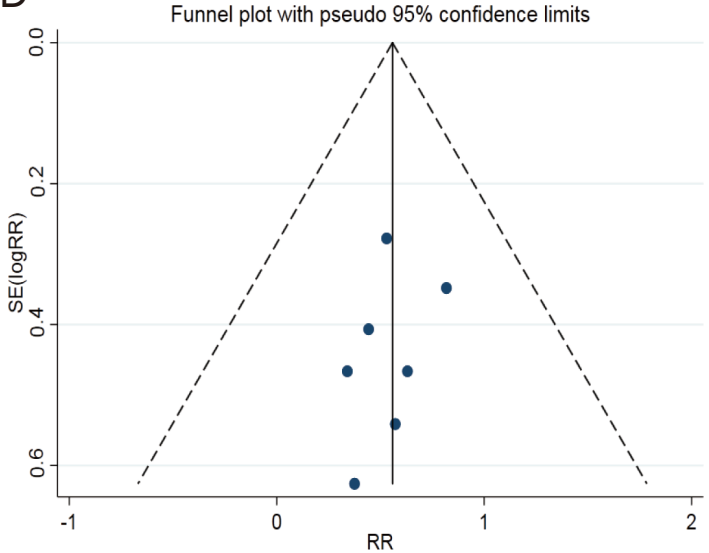

Figure 6 Funnel plots of APACHE II, PT, platelet count, and 28-day mortality. (A) Acute Physiology And Chronic Health Evaluation II, (B) platelet count, (C) prothrombin time, (D) 28-day mortality. APACHE II, Acute Physiology And Chronic Health Evaluation II; PT, platelet count. 
A

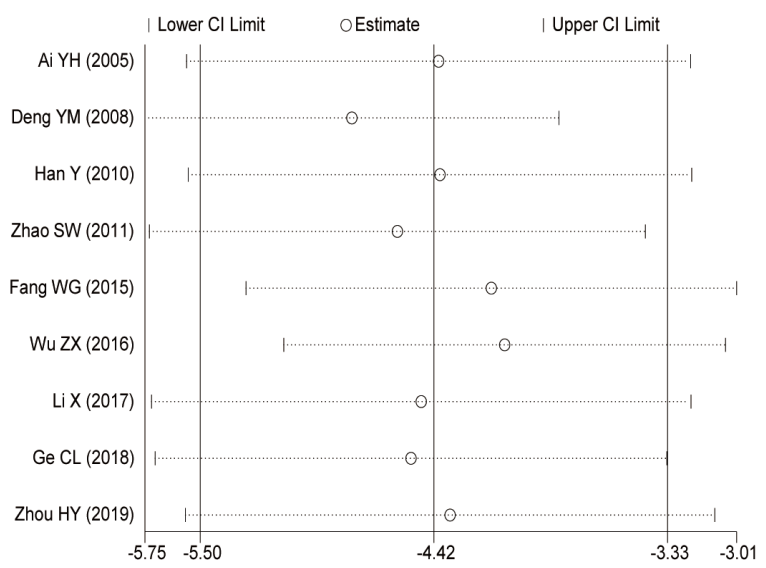

C

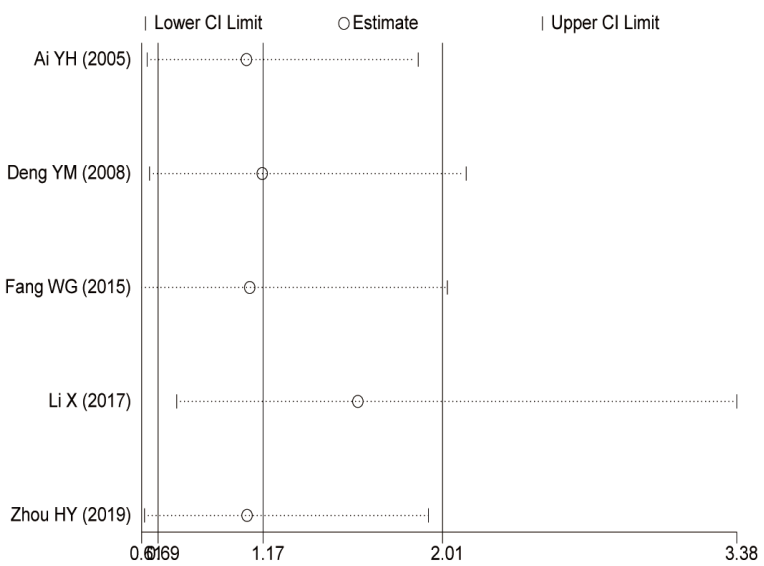

B
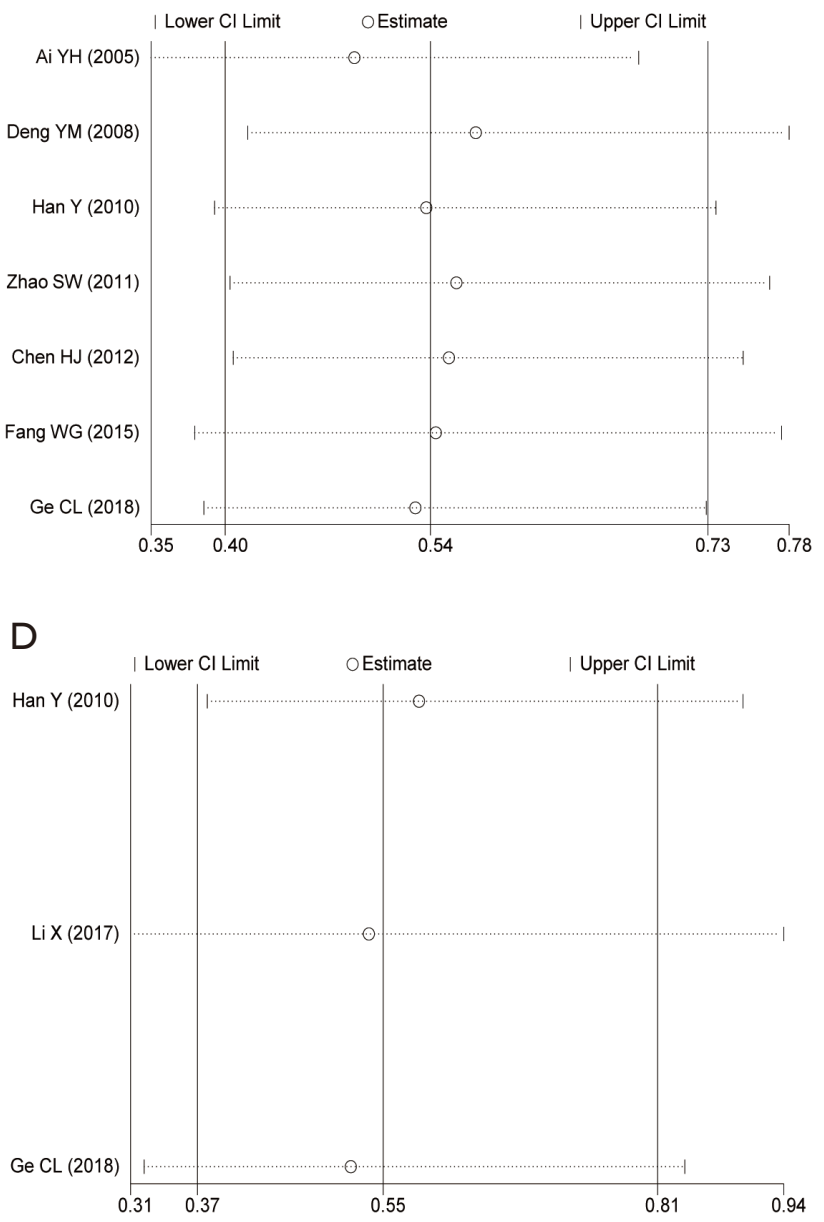

Figure 7 Sensitivity analysis of APACHE II score, 28-day mortality rate, hemorrhagic complications, and MODS. (A) Acute Physiology And Chronic Health Evaluation II (APACHE II) score, (B) 28-day mortality rate, (C) hemorrhagic complications, (D) multiple organ dysfunction syndrome. CI, confidence interval; APACHE II, Acute Physiology And Chronic Health Evaluation II; MODS, multiple organ dysfunction syndrome.

on PT, TNF- $\alpha$, and IL-6, respectively, the results of the meta-analysis changed from the original conclusion and were found to have no statistical significance.

\section{Discussion}

Sepsis is an organ dysfunction that may lead to uncontrolled lethal reaction under the infection of pathogenic microorganism. It has a complicated pathophysiological change process. The whole course of the disease involves various abnormal changes, such as inflammation, coagulation function, immune function, and microcirculation (28). Uncontrolled inflammatory response is the cornerstone of the occurrence and development of sepsis, and is currently recognized as the most important pathogenesis of sepsis (29). The course of sepsis involves cytokine production, imbalance of pro-inflammatory and anti-inflammatory reactions, overactivation of the body's immune system, and immune dysfunction. In the early stage, the production of TNF- $\alpha$ and IL- 1 jointly launch an inflammatory reaction, and a large number of inflammatory mediators increases. Of these inflammatory mediators, TNF- $\alpha$ causes bacterial endotoxin damage to the body, and its serum level has a direct impact on the prognosis of 
A

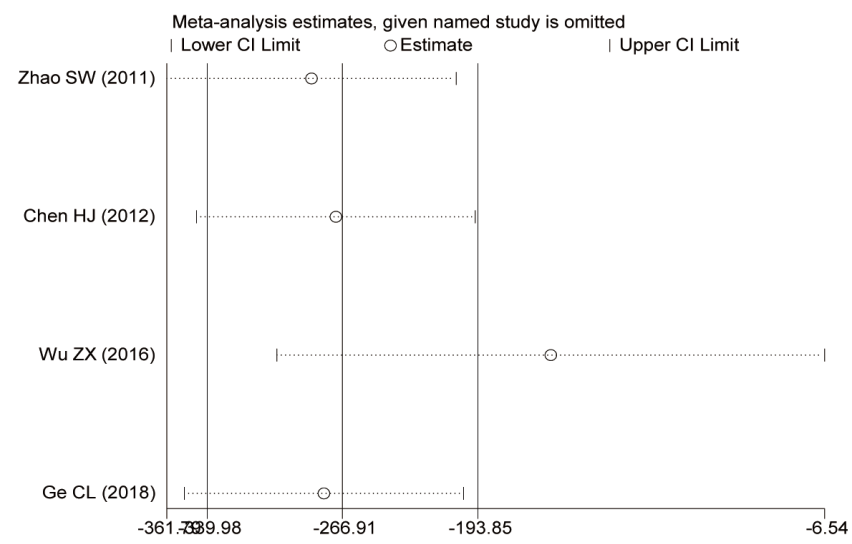

B

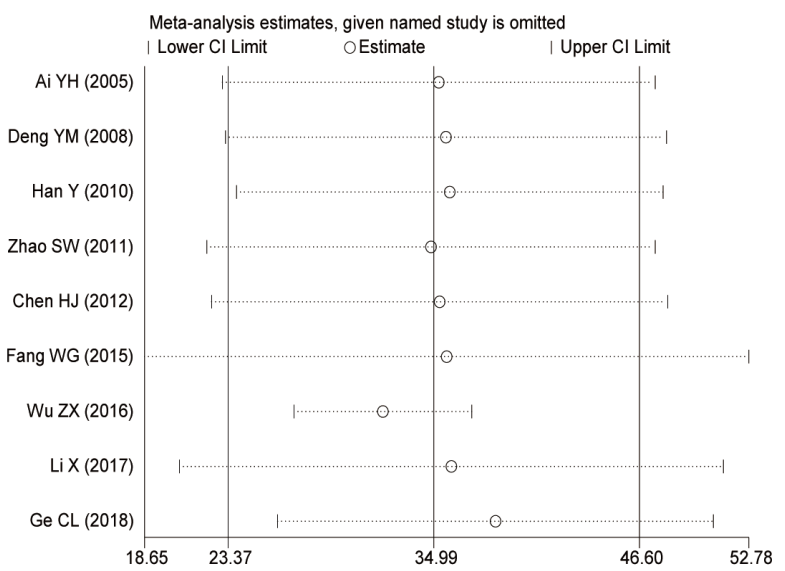

C

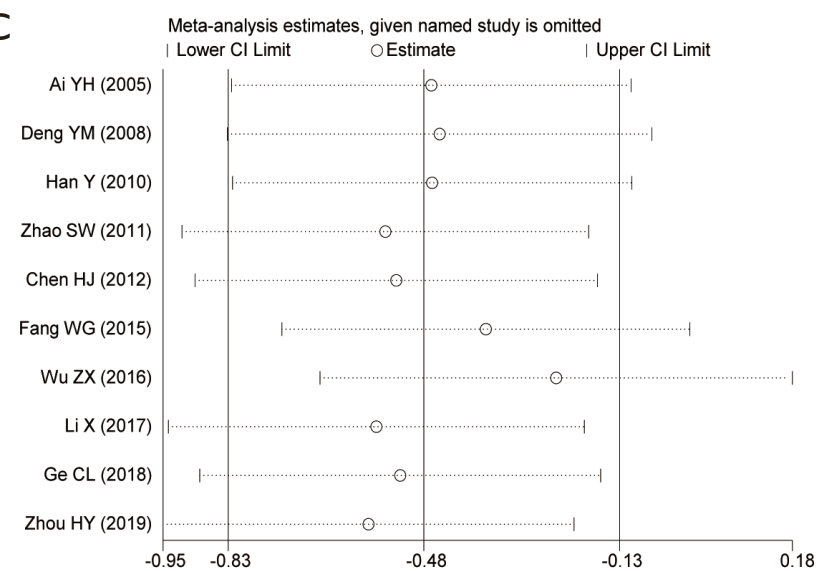

D

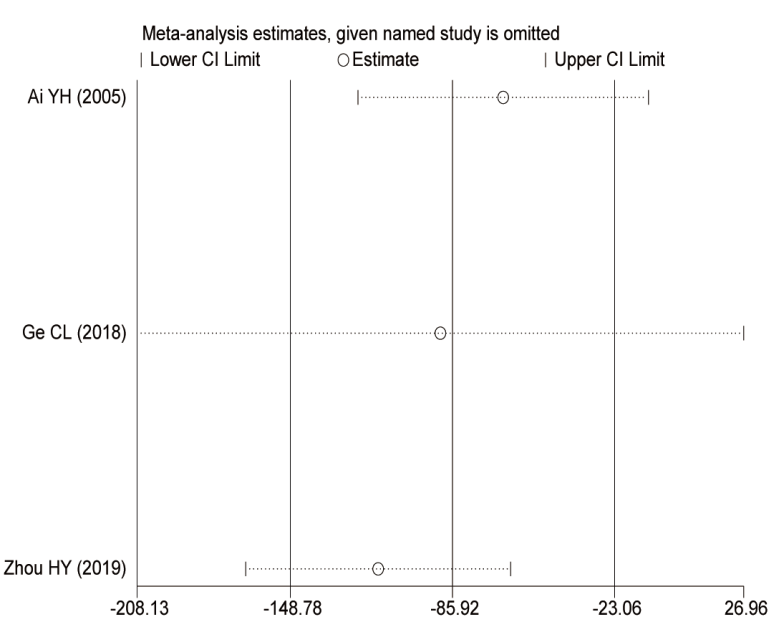

E

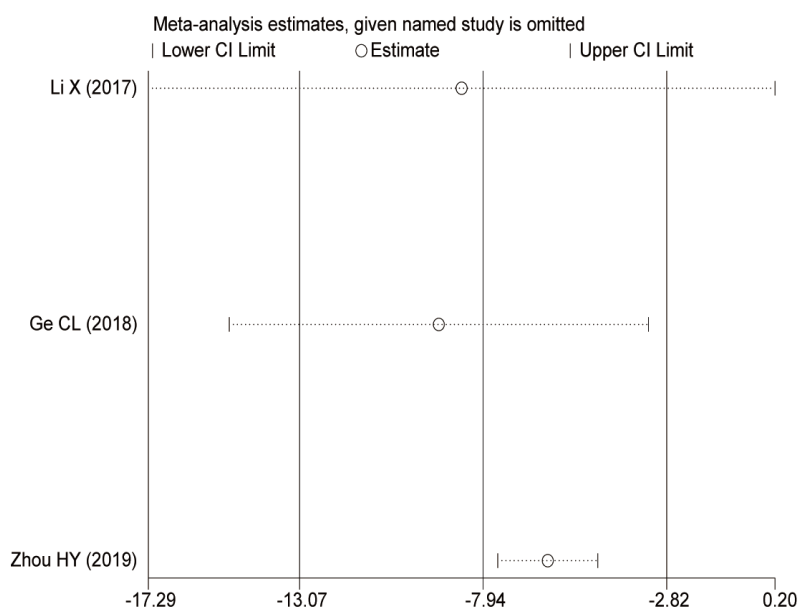

Figure 8 Sensitivity analysis of anticoagulant activity and serum inflammatory factors. (A) D-dimer, (B) platelet count, (C) prothrombin time, (D) interleukin-6, (E) tumor necrosis factor- $\alpha$. CI, confidence interval; MD, mean difference. 
patients with severe sepsis (30). IL-6 is induced by the above 2 cytokines. $\mathrm{T}$ lymphocytes proliferate and B lymphocytes produce and release immunoglobulin, both under the action of IL-6. Under the action of IL-6, the phagocytosis process of aging neutrophils will slow down, causing a substantial increase in the concentration of inflammatory mediators. Therefore, IL-6 can also be used to judge the severity and prognosis of sepsis patients. If the concentration of IL-6 increases significantly, the prognosis of the patient is often poor (31). The results of this meta-analysis show that, compared with conventional treatment, the LMWH group can significantly reduce APACHE II score, 28-day mortality rate, and MODS incidence rate with statistically significant differences. No obvious publication bias was found in the present study. Sensitivity analysis results showed that the conclusion was also robust. The LMWH group can significantly reduce the serum TNF- $\alpha$ and IL-6 levels of cytokines, but sensitivity analysis results show that these two indicators are not robust to a certain extent, which may be limited to the sample size and must be verified with a larger sample size.

After the vascular endothelium of septic patients is damaged, platelets, which should be in a quiet state in normal blood circulation, are also activated rapidly, adhere to the damaged parts of vascular endothelium and release various coagulation factors. At the same time, platelet activation factors are also released, resulting in the continuous activation of platelets, coagulation dysfunction, extensive microthrombosis, massive depletion of platelets and coagulation factors, and the formation of disseminated intravascular coagulation (32). After being activated, platelets can cause the aggregation and activation of leukocytes and neutrophils, promote the aggregation of platelets, and produce cytokines and coagulation factors, which further aggravate the body's inflammatory response and vascular damage. At the same time, the physiological anticoagulation mechanism of the patient is inhibited. Although the fibrinolytic system can be activated by tissue plasminogen activator released by endothelial cells in the early stage, the level of plasminogen activator inhibitor-1 in blood gradually increases with the progress of the disease, and the activity of the plasminogen system is also inhibited (33). The number of platelets in septic patients often decreases. The findings of the meta-analysis indicate that, compared with the conventional treatment group, the LMWH group can significantly increase platelet count and reduce PT and serum D-dimer level with statistically significant differences. There was no obvious publication bias for these three indicators. Sensitivity analysis results demonstrated that the results of the platelet count and D-dimer level were robust, but PT was not robust to a certain extent. Therefore, more studies are needed to confirm the conclusion that LMWH significantly reduces PT compared with conventional treatment.

LMWH is decomposed and purified from UFH. Its antithrombotic effect is fast and lasting, and it has been widely used in clinical settings (34). Compared with UFH, LMWH has the following advantages: (I) it has a stronger anti-factor $\mathrm{Xa}$ effect and does not need to closely monitor factor Xa; (II) it has high bioavailability and a long halflife; (III) it has less dependence on antithrombin III; and (IV) it has low probability of thrombocytopenia and less spontaneous hemorrhage (35). The results of our metaanalysis show that there was no difference in bleeding complications between the LMWH group and conventional treatment group, and the sensitivity analysis also showed that this conclusion was relatively robust. However, publication bias results indicated that there was publication bias in the study of bleeding complications. Therefore, more research is needed to confirm the effect of $\mathrm{LMWH}$ on bleeding complications. Fan et al.'s meta-analysis showed that, compared with the conventional treatment group, the level of serum D-dimer in septic patients was not reduced in the LMWH group and bleeding events increased (RR: 3.82, 95\% CI: 1.81-8.08) (36). This is different from our research results. Our results showed that the level of serum D-dimer in septic patients was significantly reduced in the $\mathrm{LMWH}$ group and did not increase bleeding events. Our sensitivity analysis results also confirmed this. The reason for this may be the difference in the choice of study. It can be seen from the results that the heterogeneity of each index in the present study was relatively low. Therefore, to a certain extent, the conclusion of the study was significant.

The present study has some limitations. First, all studies were conducted in China and published in Chinese. The results of Egger's test of bleeding complications and IL-6 were $\mathrm{P}<0.05$, which could not exclude the possibility of potential publication bias. Second, the methodological quality of some studies was not high, because it is difficult to complete blind trials in actual clinical treatment. Third, significant heterogeneity was found in the summary analysis of cytokines IL- 6 and TNF- $\alpha$, and only 3 studies were included, which had certain limitations. Finally, all of the articles only included Chinese patients, and caution should be taken when extending the results to other ethnic groups.

In conclusion, on the basis of conventional treatment, 
LMWH can reduce inflammatory reaction and improve coagulation function of adult septic patients without increasing bleeding events, significantly reduce MODS incidence rate and 28-day mortality rate, and improve prognosis. Although our research results show that LMWH will not increase bleeding events, due to certain publication bias, it is still recommended to monitor bleeding events for the safety of patients. Considering the limitations of this study, further high-quality, clinical, randomized controlled trials are needed to verify the effect of LMWH on the efficacy of adult septic patients.

\section{Acknowledgments}

Funding: None.

\section{Footnote}

Reporting Checklist: The authors have completed the PRISMA reporting checklist. Available at http://dx.doi. org/10.21037/apm-21-169

Conflicts of Interest: All authors have completed the ICMJE uniform disclosure form (available at http://dx.doi. org/10.21037/apm-21-169). The authors have no conflicts of interest to declare.

Ethical Statement: The authors are accountable for all aspects of the work in ensuring that questions related to the accuracy or integrity of any part of the work are appropriately investigated and resolved.

Open Access Statement: This is an Open Access article distributed in accordance with the Creative Commons Attribution-NonCommercial-NoDerivs 4.0 International License (CC BY-NC-ND 4.0), which permits the noncommercial replication and distribution of the article with the strict proviso that no changes or edits are made and the original work is properly cited (including links to both the formal publication through the relevant DOI and the license). See: https://creativecommons.org/licenses/by-nc-nd/4.0/.

\section{References}

1. Mayr FB, Yende S, Angus DC. Epidemiology of severe sepsis. Virulence 2014;5:4-11.

2. Martin GS, Mannino DM, Eaton S, et al. The Epidemiology of Sepsis in the United States from 1979 through 2000. N Engl J Med 2003;348:1546-54.

3. Claushuis TA, de Stoppelaar SF, Stroo I, et al. Thrombin contributes to protective immunity in pneumonia-derived sepsis via fibrin polymerization and platelet-neutrophil interactions. J Thromb Haemost 2017;15:744-57.

4. Cotovio M, Monreal L, Armengou L, et al. Fibrin deposits and organ failure in newborn foals with severe septicemia. J Vet Intern Med 2008;22:1403-10.

5. Yamakawa K, Gando S, Ogura H, et al. Identifying Sepsis Populations Benefitting from Anticoagulant Therapy:

A Prospective Cohort Study Incorporating a Restricted Cubic Spline Regression Model. Thromb Haemost 2019;119:1740-51.

6. Umemura Y, Yamakawa K. Optimal patient selection for anticoagulant therapy in sepsis: an evidence-based proposal from Japan. J Thromb Haemost 2018;16:462-4.

7. Zarychanski R. From mice to men: Treating sepsis with heparin. Crit Care Med 2011;39:1225-6.

8. Posma JJ, Grover SP, Hisada Y, et al. Roles of Coagulation Proteases and PARs (Protease-Activated Receptors) in Mouse Models of Inflammatory Diseases. Arterioscler Thromb Vasc Biol 2019;39:13-24.

9. Day JR, Taylor KM, Lidington EA, et al. Aprotinin inhibits proinflammatory activation of endothelial cells by thrombin through the protease-activated receptor $1 . \mathrm{J}$ Thorac Cardiovasc Surg 2006;131:21-7.

10. Cornet AD, Smit EG, Beishuizen A, et al. The role of heparin and allied compounds in the treatment of sepsis. Thromb Haemost 2007;98:579-86.

11. Dellinger RP, Levy MM, Rhodes A, et al. Surviving sepsis campaign: international guidelines for management of severe sepsis and septic shock: 2012. Crit Care Med 2013;41:580-637.

12. Kahn SR, Lim W, Dunn AS, et al. Prevention of VTE in Nonsurgical Patients. Chest 2012;141:e195S-e226S.

13. Radulovic V, Hyllner M, Ternström L, et al. Sustained heparin effect contributes to reduced plasma thrombin generation capacity early after cardiac surgery. Thromb Res 2012;130:769-74.

14. Renda G, Di Pillo R, D'Alleva A, et al. Surgical bleeding after pre-operative unfractionated heparin and low molecular weight heparin for coronary bypass surgery. Haematologica 2007;92:366-73.

15. Bucci C, Geerts WH, Sinclair A, et al. Comparison of the effectiveness and safety of low-molecular weight heparin versus unfractionated heparin anticoagulation after heart valve surgery. Am J Cardiol 2011;107:591-4.

16. Incampo F, Carrieri C, Galasso R, et al. Co-administration 
of low molecular weight heparin enhances the profibrinolytic effect of warfarin through different mechanisms. Thromb Res 2014;133:634-9.

17. Higgins JP, Thompson SG, Deeks JJ, et al. Measuring inconsistency in meta-analyses. BMJ 2003;327:557-60.

18. Ai YH, Zhang LN, Gong H, et al. Clinical study of low molecular weight heparin therapy for sepsis. Zhongguo Wei Zhong Bing Ji Jiu Yi Xue 2005;17:736-9.

19. Deng YM, Fan XD, Xue XY, et al. Clinical study of low molecular weight heparin therapy for sepsis. Chin J Emerg Med 2008;17:132-6.

20. Han Y, Chen QH, Kuang CS, et al. Study of changes and clinical significance of $\mathrm{P}$-selectin in low molecular weight heparin therapy for septic patients. Chin J Crit Care Med 2010;30:976-8.

21. Zhao SW, Chai YF. The influence of Xuebijing injection and low molecular weight heparin on coagulation function and prognosis in patients with severe sepsis. Chin J Emerg Med 2011;20:405-8.

22. Chen HJ. Study on Change of Coagulation and Treatment in Severe Patients. Practical Journal of Cardiac Cerebral Pneumal and Vascular Disease 2012;20:1990-1.

23. Fang W. Clinical effects of low molecular weight heparin on sepsis. Journal of Clinical Emergency 2015;16:411-5.

24. Wu ZH, Luo ZC, Tan JY, et al. Analysis of low molecular weight heparin sodium for the treatment of 30 cases of patients with sepsis. Shanghai Medical \& Pharmaceutical Journal 2016;37:36-42.

25. Li X, Zheng XJ, Cui W, et al. Effects of low molecular weight heparin on TNF- $\alpha$, IL- 6 and coagulation in patients with sepsis. Chinese Journal of Difficult and Complicated Cases 2017;16:566-9.

26. Ge CL, Song R. Early application of low molecular heparin in treatment of senile severe sepsis. Clin Focus 2018;33:153-6.

27. Zhou HY, Tan J, Gai L, et al. Effect of Molecular Weight Heparin on Oxygenation Index and Inflammatory Reaction of Sepsis Patients with ALI/ARDS in ICU. China World
Econ 2019;22:106-8.

28. Wang Y, Braun OÖ, Zhang S, et al. Monocytes regulate systemic coagulation and inflammation in abdominal sepsis. American journal of physiology. Heart and circulatory physiology 2015;308:H540-7.

29. Liu AP, Yuan QH, Zhang B, et al. Cannabinoid receptor 2 activation alleviates septic lung injury by promoting autophagy via inhibition of inflammatory mediator release. Cell Signal 2020;69:109556.

30. Drewry AM, Ablordeppey EA, Murray ET, et al. Comparison of monocyte human leukocyte antigen-DR expression and stimulated tumor necrosis factor alpha production as outcome predictors in severe sepsis: a prospective observational study. Crit Care 2016;20:334.

31. Song J, Park DW, Moon S, et al. Diagnostic and prognostic value of interleukin-6, pentraxin 3, and procalcitonin levels among sepsis and septic shock patients: a prospective controlled study according to the Sepsis-3 definitions. BMC Infect Dis 2019;19:968.

32. Yamakawa K, Yoshimura J, Ito T, et al. External Validation of the Two Newly Proposed Criteria for Assessing Coagulopathy in Sepsis. Thromb Haemost 2019;119:203-12.

33. Levi M, Poll TV. Coagulation in patients with severe sepsis. Semin Thromb Hemost 2015;41:9-15.

34. Imberti D, Cimminiello C, Di Nisio M, et al. Antithrombotic therapy for venous thromboembolism in patients with cancer: expert guidance. Expert Opin Pharmacother 2018;19:1177-85.

35. Batta A, Kalra BS, Khirasaria R. Critical Issues and Recent Advances in Anticoagulant Therapy: A Review. Neurol India 2019;67:1200-12.

36. Fan $Y$, Jiang $M$, Gong D, et al. Efficacy and safety of low-molecular-weight heparin in patients with sepsis: a meta-analysis of randomized controlled trials. Sci Rep 2016;6:25984.

(English Language Editor: R. Scott)
Cite this article as: Li X, Liu Z, Luo M, Xi Y, Li C, Wang S, Yang R. Therapeutic effect of low-molecular-weight heparin on adult sepsis: a meta-analysis. Ann Palliat Med 2021;10(3):31153127. doi: 10.21037/apm-21-169 